\title{
Opérateurs de Riesz dont le coeur analytique est fermé
}

\author{
par \\ Widad Bouamama (Lille)
}

Résumé. Dans ce travail nous donnons plusieurs caractérisations, en termes spectraux, d'opérateurs de Riesz dont le coeur analytique est fermé. Notamment, nous montrons que pour un opérateur de Riesz $T$, le coeur analytique est fermé si et seulement si sa dimension est finie si et seulement si zéro est isolé dans le spectre de $T$ si et seulement si $T=Q+F$ avec $Q F=F Q=0, F$ de rang fini et $Q$ quasinilpotent. Ce dernier résultat montre qu'un opérateur de Riesz dont le coeur analytique est fermé admet la décomposition de West. D'autre part, plusieurs conditions équivalentes sont données pour que zéro soit un pôle d'ordre fini de la résolvente de $T$.

1. Préliminaires. Soient $X$ un espace de Banach complexe et $B(X)$ l'algèbre des opérateurs linéaires bornés de $X$ dans lui-même. Si $T \in B(X)$, on note $N(T), R(T)$ et $\sigma(T)$ respectivement le noyau, l'image et le spectre de $T$. On notera également $\operatorname{nul}(T)=\operatorname{dim} N(T)$ et $\operatorname{def}(T)=\operatorname{codim} R(T)$.

L'opérateur $T$ est dit de Fredholm si l'image de $T$ est fermée et $\max \{\operatorname{nul}(T), \operatorname{def}(T)\}$ est fini. Dans ce cas l'indice de $T$ sera noté $\operatorname{ind}(T)$; par définition, $\operatorname{ind}(T)=\operatorname{nul}(T)-\operatorname{def}(T)$.

$\mathcal{K}(X)$ désignera l'idéal fermé dans $B(X)$ des opérateurs compacts. Soit $C(X)=B(X) / \mathcal{K}(X)$ l'algèbre de Calkin et soit $\pi: B(X) \rightarrow C(X)$ la surjection canonique.

L'ensemble des opérateurs de Fredholm sera noté $\Phi(X)$. Par le théorème d'Atkinson on a

$$
T \in \Phi(X) \Leftrightarrow \pi(T) \text { est inversible dans } C(X) .
$$

La partie quasinilpotente de $T$ sera notée par

$$
H_{0}(T)=\left\{u \in X ; \lim _{n \rightarrow \infty}\left\|T^{n} u\right\|^{1 / n}=0\right\} .
$$

Elle a été introduite dans [13, p. 418], dans le cas où 0 est un point isolé du spectre de $T$.

2000 Mathematics Subject Classification: 47A53, 47A68, 46B04.

Key words and phrases: opérateur de Riesz, coeur analytique, partie quasinilpotente d'un opérateur. 
Soit $K(T)$ le coeur analytique défini par $K(T)=\left\{u \in X ; \exists a>0 \forall n \geq 0 \exists v_{n} \in X\right.$ vérifiant :

$$
\begin{aligned}
& \text { (1) } v_{0}=u \text { et } T v_{n+1}=v_{n}, \\
& \text { (2) } \left.\left\|v_{n}\right\| \leq a^{n}\|u\| \text { pour tout } n \geq 0\right\} .
\end{aligned}
$$

Notons que la condition (2) de la définition de $K(T)$ peut être remplacée par $\lim \sup _{n \rightarrow \infty}\left\|v_{n}\right\|^{1 / n}<\infty$.

Les sous-espaces $H_{0}(T)$ et $K(T)$ ont été introduits dans [11] et étudiés dans $[2,5,8,11-15]$. Les deux lemmes suivants regroupent quelques propriétés des sous-espaces $H_{0}(T)$ et $K(T)$ dans le cas où $T$ est un opérateur borné (voir $[11-13,15]$ ).

Lemme 1.1. Soit $T$ un opérateur borné. Alors :

(i) $H_{0}(T)$ est un sous-espace non nécessairement fermé de $X$.

(ii) Pour tout $j \geq 0, N\left(T^{j}\right) \subseteq H_{0}(T)$.

(iii) $x \in H_{0}(T) \Leftrightarrow T x \in H_{0}(T)$.

(iv) $H_{0}(T)=X \Leftrightarrow T$ est quasinilpotent (i.e. $\sigma(T)=\{0\}$ ).

Lemme 1.2. Soit $T$ un opérateur borné. Alors :

(1) $K(T)$ est un sous-espace non nécessairement fermé de $X$.

(2) $T(K(T))=K(T)$.

(3) Si $X_{0}$ est un sous-espace fermé de $X$ avec $T\left(X_{0}\right)=X_{0}$ alors $X_{0} \subseteq$ $K(T)$.

(4) Si T est quasinilpotent alors $K(T)=\{0\}$.

Pour la preuve du théorème suivant voir $[11,15,8]$.

ThÉorème 1.3. Soit $T \in B(X)$. Alors $\lambda_{0}$ est un point isolé dans $\sigma(T)$ si et seulement si $X=K\left(T-\lambda_{0} I\right) \oplus H_{0}\left(T-\lambda_{0} I\right)$ et $H_{0}\left(T-\lambda_{0} I\right) \neq\{0\}$ $\left(\oplus\right.$ désigne la somme directe topologique) si et seulement si $K\left(T-\lambda_{0} I\right)$ est fermé et $X=K\left(T-\lambda_{0} I\right) \oplus H_{0}\left(T-\lambda_{0} I\right)$ et $H_{0}\left(T-\lambda_{0} I\right) \neq\{0\} \quad$ (ici $\oplus$ désigne la somme directe algébrique).

De plus, $\lambda_{0}$ est pôle d'ordre fini de la résolvente de $T$ si et seulement s'il existe d entier tel que $H_{0}\left(T-\lambda_{0} I\right)=N\left(T-\lambda_{0} I\right)^{d}$ et $K\left(T-\lambda_{0} I\right)=$ $R\left(T-\lambda_{0} I\right)^{d}$. Dans ce cas $X=N\left(T-\lambda_{0} I\right)^{d} \oplus R\left(T-\lambda_{0} I\right)^{d}$.

\section{Résultats}

DÉfinition. $T \in B(X)$ est dit opérateur de Riesz (et on note $T \in \mathcal{R}(X)$ ) si pour tout $\lambda \in \mathbb{C} \backslash\{0\}, T-\lambda I$ est de Fredholm.

Autrement dit, par (1.1), $T \in \mathcal{R}(X)$ si et seulement si $T$ est quasinilpotent dans l'algébre de Calkin ( $\Leftrightarrow$ le spectre essentiel $\sigma_{\mathrm{e}}(T)=\sigma(\pi(T))=$ $\{0\})$. On a $\mathcal{K}(X) \subset \mathcal{R}(X)$. Pour plus de détails sur les opérateurs de Riesz voir $[1,3,4]$. 
Remarque. Pour un opérateur $T \in \mathcal{R}(X)$, on a :

(1) $0 \in \sigma(T)$ et $\sigma(T)$ est au plus dénombrable ayant pour seul point d'accumulation eventuel zéro.

(2) $\forall \lambda \neq 0$, ind $(T-\lambda I)=0$. En particulier pour $\lambda \neq 0$,

$$
N(T-\lambda I)=0 \Leftrightarrow T-\lambda I \text { inversible. }
$$

(3) Si $\lambda \in \sigma(T) \backslash\{0\}$ alors $\lambda$ est isolé dans le spectre de $T$, en plus la projection spectrale associée à l'ensemble spectral $\{\lambda\}$ est de rang fini.

Pour la preuve du lemme suivant, on a besoin de la théorie spectrale locale; nous renvoyons à la référence [9].

Lemme 2.1. Si $T \in \mathcal{R}(X)$ alors $H_{0}(T)$ est fermé.

Démonstration. Puisque les opérateurs de Riesz ont la propriété de Dunford $(\mathrm{C}), H_{0}(T)=\left\{x \in X ; \sigma_{T}(x) \subseteq\{0\}\right\}$ est fermé (voir [9, pp. 27 et 42]), où $\sigma_{T}(x)$ est le spectre local de $T$ en $x$.

Remarque. La condition " $H_{0}(T)$ fermé" n'est pas sufisante pour que 0 soit isolé dans $\sigma(T)$ : nous démontrerons dans le théorème 2.3 qu'il faut en plus que codim $H_{0}(T)$ soit finie.

Lemme 2.2. Soit $T \in B(X)$, et supposons qu'il existe $Q$ et $F$ dans $B(X)$ tels que $T=Q+F, Q$ quasinilpotent et $F Q=Q F=0$. Alors :

(1) $H_{0}(T)=H_{0}(F)$.

(2) Si $F$ est de rang fini, alors codim $H_{0}(T)$ est finie aussi.

Démonstration. (1) $Q F=F Q=0$ implique que pour tout $n \geq 1$,

$$
T^{n}=Q^{n}+F^{n} \text {. }
$$

Soient $x \in H_{0}(F)$ et $\varepsilon>0$. Alors il existe $N \in \mathbb{N}$ tel que pour tout $n \geq N$, $\left\|F^{n} x\right\| \leq(\varepsilon / 2)^{n}$ et $\left\|Q^{n} x\right\| \leq(\varepsilon / 2)^{n}$ ( $Q$ étant quasinilpotent). D'où, en utilisant (2.1), $\left\|T^{n} x\right\| \leq\left\|Q^{n} x\right\|+\left\|F^{n} x\right\| \leq 2(\varepsilon / 2)^{n}$, ce qui implique que $\left\|T^{n} x\right\|$ converge vers zéro. Par conséquent, $x \in H_{0}(T)$ et donc $H_{0}(F) \subseteq$ $H_{0}(T)$.

Puisque, par (2.1), $F^{n}=T^{n}-Q^{n}$ pour tout $n \geq 1$, en utilisant le même raisonnement que plus haut, on obtient $H_{0}(T) \subset H_{0}(F)$.

(2) Supposons que $F$ est de rang fini et soit l'application $\widehat{F}: X / N(F) \rightarrow$ $R(F)$. Alors $\widehat{F}$ est une bijection. D'où $\operatorname{codim} N(F)=\operatorname{dim}(X / N(F))=$ $\operatorname{dim} R(F)$ est finie. Il résulte alors que la codimension de $H_{0}(T)$ est finie, puisque $N(F) \subset H_{0}(F)=H_{0}(T)$.

Remarque. Dans [5], et pour un opérateur $T$ compact, W. Gong et L. Wang démontrent le résultat suivant :

$K(T)$ est fermé $\Leftrightarrow 0$ est isolé dans $\sigma(T) \Leftrightarrow K(T)$ est de dim finie $\Leftrightarrow \sigma(T)$ est fini. 
En utilisant des preuves analogues, il est facile de vérifier que ces équivalences sont également vraies pour les opérateurs de Riesz.

Dans ce papier, nous allons démontrer que si $T$ est un opérateur de Riesz, ces propriétés sont aussi équivalentes au fait que codim $H_{0}(T)$ est finie.

ThÉorème 2.3. Soit $T \in B(X)$ un opérateur de Riesz. Alors les conditions suivantes sont équivalentes:

(1) $K(T)$ est fermé.

(2) $T=Q+F$, où $Q, F \in B(X)$, avec $Q F=F Q=0, \sigma(Q)=\{0\}$ et $F$ de rang fini.

(3) $\operatorname{codim} H_{0}(T)$ est finie.

(4) $\sigma(T)$ est un ensemble fini.

(5) $K\left(T^{*}\right)$ est fermé.

(6) codim $H_{0}\left(T^{*}\right)$ est finie.

Démonstration. $(1) \Rightarrow(2)$. $K(T)$ étant fermé, d'après la remarque précédente, on déduit que 0 est isolé dans $\sigma(T)$, il existe alors deux sous-espaces $X_{1}$ et $X_{2}$ fermés de $X$ invariants par $T$ et tels que $X=X_{1} \oplus X_{2}$. Soit $T_{i}=T_{\mid X_{i}}, i=1,2$. Alors $\sigma\left(T_{1}\right)=\{0\}$. Soit $P$ la projection sur $X_{1}$. Alors $P$ commute avec $T$ et puisque $T \in \mathcal{R}(X), I-P$ est de rang fini. D'autre part, on a $\left\|T^{n} P\right\|^{1 / n}=\left\|T_{1}^{n}\right\|^{1 / n}$, qui tend vers 0 . Donc si on pose $Q=T P$ et $F=(I-P) T$, alors $T=Q+F, F$ est de rang fini, $Q$ est quasinilpotent et $Q F=F Q=0$.

$(2) \Rightarrow(3)$. Voir Lemme 2.2 .

$(3) \Rightarrow(4)$. Puisque $T \in \mathcal{R}(X), H_{0}(T)$ est fermé (voir Lemme 2.1). Supposons que codim $H_{0}(T)$ est finie; alors il existe $M$ sous-espace de $X$, de dimension finie tel que $X=H_{0}(T) \oplus M$. Soit $T=\left(\begin{array}{cc}T_{0} & A \\ 0 & T_{1}\end{array}\right)$ la représentation matricielle de $T$ suivant cette décomposition. Alors, $\sigma\left(T_{0}\right)=\{0\}$ par le Lemme 1.1(iv) et $\sigma\left(T_{1}\right)$ est fini puisque $\operatorname{dim} M<\infty$. Or $\sigma(T) \subseteq \sigma\left(T_{0}\right) \cup$ $\sigma\left(T_{1}\right)$. D'où $\sigma(T)$ est un ensemble fini.

$(4) \Rightarrow(1)$. Supposons que $\sigma(T)=\{0\} \cup\left\{\lambda_{1}, \ldots, \lambda_{n}\right\}$ est un ensemble fini. Puisque $T \in \mathcal{R}(X)$, la projection spectrale associée à l'ensemble spectral $\left\{\lambda_{1}, \ldots, \lambda_{n}\right\}$ est de rang fini. On en déduit que $X=H_{0}(T) \oplus M$, avec la dimension de $M$ finie. D'autre part, 0 étant isolé dans $\sigma(T)$, le Théorème 1.3 implique que $X=H_{0}(T) \oplus K(T)$; il résulte alors que $\operatorname{dim} K(T)=$ codim $H_{0}(T)=\operatorname{dim} M$ est finie et donc $K(T)$ est fermé.

On a donc les équivalences entre (1), (2), (3) et (4); pour vérifier celles de (5) et (6), il suffit de remarquer que si $T$ est de Riesz, alors $T^{*}$ l'est aussi, et si 0 est isolé dans $\sigma(T)$, alors il l'est aussi dans $\sigma\left(T^{*}\right)$, et d'appliquer les mêmes arguments que précédemment.

Comme conséquence directe du théorème précédent, on a le corollaire suivant : 
Corollaire 2.4. Soit $T \in \mathcal{R}(X)$ avec $K(T)$ fermé. Alors il existe $Q \in$ $B(X)$ quasinilpotent et $K \in B(X)$ compact tel que

$$
T=Q+K \text {. }
$$

En fait, $K$ est de rang fini et $Q K=K Q=0$.

REMARQUE. Le corollaire précédent donne une réponse partielle positive à la question encore ouverte suivante, concernant le relèvement des opérateurs quasinilpotents.

Question. Si $T \in B(X), X$ espace de Banach de dimension infinie, quasinilpotent dans l'algèbre de Calkin (i.e. $T \in \mathcal{R}(X)$ ), a-t-on $T=Q+K$, où $Q \in B(X)$ est quasinilpotent et $K \in B(X)$ est compact ?

Rappelons que si $X=H$ est un espace de Hilbert alors la réponse à cette question est positive. Ce résultat est dû à T. T. West [17] (voir aussi [4]).

DÉfinition. Pour $T \in B(X)$, on appelle ascente de $T$ et on note $\operatorname{asc}(T)$ le plus petit entier $p$ tel que $N\left(T^{p}\right)=N\left(T^{p+1}\right)$; si un tel entier n'existe pas alors $\operatorname{asc}(T)=\infty$.

On appelle descente de $T$ et on note $\operatorname{desc}(T)$ le plus petit entier $q$ tel que $R\left(T^{q}\right)=R\left(T^{q+1}\right)$; si un tel entier n'existe pas alors $\operatorname{desc}(T)=\infty$.

Remarque. Pour un exposé détaillé sur l'ascente et la descente d'un opérateur on peut consulter $[16, \mathrm{Ch} . \mathrm{V}]$.

Un opérateur $T \in B(X)$ est dit méromorphique si pour tout $\lambda \in \mathbb{C} \backslash\{0\}$, $\max \{\operatorname{asc}(T-\lambda I) ; \operatorname{desc}(T-\lambda I)\}<\infty$. Ceci est équivalent à dire que tout $\lambda \in \mathbb{C} \backslash\{0\}$ est un pôle d'ordre fini de la résolvente de $T$.

Il est facile de voir que si $T$ est un opérateur de Riesz alors $T$ est méromorphique (voir [16]). Par contre, un opérateur méromorphique n'est pas toujours de Riesz : comme contre-exemple, il suffit de considérer une projection bornée dont le noyau est de dimension infinie. Elle est bien sur d'ascente et de descente finies, mais n'est pas de Riesz.

Par un raisonnement analogue à celui utilisé dans la preuve du Théorème 2.3, on obtient sans difficulté le résultat suivant :

ThÉorème 2.5. Soit $T \in B(X)$ un opérateur meromorphique. Alors les conditions suivantes sont équivalentes :

(1) $K(T)$ est fermé.

(2) $0 \in \sigma(T)$ est isolé.

(3) $T=Q+F$, où $Q, F \in B(X)$, avec $Q F=F Q=0, \sigma(Q)=\{0\}$ et $\sigma(F)$ fini.

(4) codim $H_{0}(T)$ est finie.

(5) $\sigma(T)$ est un ensemble fini.

De plus $\sigma(T)=\{0\} \Leftrightarrow K(T)=\{0\}$. 
Proposition 2.6. Soit $T \in B(X)$ et $d$ un entier. Alors les conditions suivantes sont équivalentes:

(i) $T^{d}=0$.

(ii) $\sigma(T)=\{0\}$ et $\operatorname{desc}(T) \leq d$.

(iii) $\sigma(T)=\{0\}, \operatorname{asc}(T) \leq d$ et $R\left(T^{d+1}\right)$ est fermé.

Démonstration. Les implications (i) $\Rightarrow$ (ii) et (i) $\Rightarrow$ (iii) sont évidentes. D'autre part, l'opérateur $T$ étant borné, $R\left(T^{d}\right)$ muni de la norme $\|y\|=$ $\|y\|+\inf _{x \in X, y=T^{d} x}\|x\|$ est un espace de Banach. Soit $T_{0}=T: R\left(T^{d}\right) \rightarrow$ $R\left(T^{d}\right)$. Alors il est facile de voir que $\sigma\left(T_{0}\right) \subseteq \sigma(T)=\{0\}$.

Montrons que (ii) implique (i). Puisque $\operatorname{desc}(T) \leq d, T_{0}$ est surjectif. Par continuité de l'indice ( $T_{0}$ étant surjectif et $\sigma\left(T_{0}\right) \subseteq\{0\}$ ), on déduit que $T_{0}$ est inversible. Par conséquent, $\sigma\left(T_{0}\right)$ est vide, ce qui implique que $R\left(T^{d}\right)=\{0\}$ et donc $T^{d}=0$.

Montrons maintenant que (iii) implique (i). Puisque asc $(T) \leq d, N\left(T_{0}\right)=$ $N(T) \cap R\left(T^{d}\right)=\{0\}$ et donc $T_{0}$ est injectif. D'autre part, $R\left(T^{d+1}\right)$ fermé implique que l'image de $T_{0}$ est fermée. Par conséquent, $T_{0}$ est injectif à l'image fermée. Par continuité de l'indice, on déduit que $T_{0}$ est inversible. Par conséquent, $\sigma\left(T_{0}\right)$ est vide, ce qui implique que $R\left(T^{d}\right)=\{0\}$ et donc $T^{d}=0$.

Remarque. Les résultats de la proposition 2.6 ont déja été obtenus dans [6] et [16], mais ici la preuve est nouvelle.

Comme conséquence directe de la proposition précédente, on a le corollaire suivant (voir $[6,15]$ ).

Corollaire 2.7. Soit $T \in B(X)$ avec $d=\operatorname{desc}(T)$ finie. Alors

$$
\sigma(T)=\{0\} \Leftrightarrow T^{d}=0 .
$$

Proposition 2.8. Soit $T \in B(X)$ avec $d=\operatorname{desc}(T)$ finie. Alors les conditions suivantes sont équivalentes:

(a) $H_{0}(T)$ est fermé.

(b) $H_{0}(T)=N\left(T^{d}\right)$.

(c) $\operatorname{asc}(T)$ est finie.

(d) Zéro est un pôle d'ordre fini de la résolvente de $T$.

Démonstration. (a) $\Rightarrow(\mathrm{b}) . H_{0}(T)$ étant fermé donc un espace de Banach, soit $T_{0}=T: H_{0}(T) \rightarrow H_{0}(T)$. Alors $\sigma\left(T_{0}\right)=\{0\}$ (voir Lemme 1.1(iii)). D'autre part, en utilisant encore le Lemme 1.1(ii), on voit facilement que

$$
R\left(T_{0}^{d}\right)=R\left(T^{d}\right) \cap H_{0}(T)=R\left(T^{d+1}\right) \cap H_{0}(T)=R\left(T_{0}^{d+1}\right) .
$$

Donc $d=\operatorname{desc}\left(T_{0}\right)$ finie. Maintenant, le Corollaire 2.7 implique que $T_{0}^{d}=0$. D'où $N\left(T^{d}\right) \subseteq H_{0}(T)=N\left(T_{0}^{d}\right) \subseteq N\left(T^{d}\right)$ et donc $H_{0}(T)=N\left(T^{d}\right)$.

(b) $\Rightarrow$ (c). Puisque $\forall j \geq 0, N\left(T^{j}\right) \subseteq H_{0}(T)=N\left(T^{d}\right)$, asc(T) est finie. 
$(\mathrm{c}) \Rightarrow(\mathrm{d})$. Conséquence directe du [15, Théorème 10.2].

$(\mathrm{d}) \Rightarrow(\mathrm{a})$. Conséquence du Théorème 1.3.

Un opérateur $T \in B(X)$ est dit algébrique si il existe un polynôme $P$ non nul tel que $P(T)=0$.

ThÉorème 2.9. Soit $T \in B(X)$ un opérateur de Riesz. Alors les conditions suivantes sont équivalentes:

(1) $0 \in \sigma(T)$ est un pôle d'ordre fini de la résolvente de $T$.

(2) $T$ est algébrique.

(3) Il existe $k \geq 0$ tel que codim $N\left(T^{k}\right)<\infty$.

(4) $j=\operatorname{asc}(T)$ est finie et $R\left(T^{j+1}\right)$ est fermé.

(5) $T=Q+F$, ò̀ $Q, F \in B(X)$, avec $Q F=F Q=0, Q$ nilpotent et $F$ de rang fini.

Remarque. Dans [5], W. Gong et L. Wang démontrent le résultat suivant : Si $T$ est un opérateur compact, alors les conditions suivantes sont équivalentes :

(1) Il existe $d \geq 0$ tel que $\operatorname{dim} R\left(T^{d}\right)<\infty$.

(2) Il existe $n \geq 0$ tel que $K(T)=R\left(T^{n}\right)$.

(3) $\operatorname{desc}(T)<\infty$.

(4) $0 \in \sigma(T)$ est un pôle d'ordre fini de la résolvente de $T$.

En utilisant les mêmes arguments, on vérifie facilement que ces équivalences restent vrais également pour les opérateurs de Riesz.

Démonstration du Théorème 2.9. (1) $\Leftrightarrow(2)$. Le Théorème 2.3 implique que $\sigma(T)$ est un ensemble fini. D'autre part, $T \in \mathcal{R}(X)$ et l'hypothèse (1) impliquent que tout $\lambda \in \sigma(T)$ est un pôle d'ordre fini de la résolvente de $T$; ceci est équivalent à $T$ algèbrique (voir [9, Th. 4.1 et Th. 4.3]).

$(1) \Rightarrow(3)$. D'aprés le Théorème 1.3 , il existe $d$ entier tel que $X=N\left(T^{d}\right) \oplus$ $R\left(T^{d}\right)$. Donc $\operatorname{dim} R\left(T^{d}\right)=\operatorname{codim} N\left(T^{d}\right)=\operatorname{codim} H_{0}(T)<\infty$ par le Théorème 2.3 .

$(1) \Rightarrow(4)$. Conséquence du Théorème 1.3.

$(3) \Rightarrow(2)$. L'application $\widehat{T^{k}}: X / N\left(T^{k}\right) \rightarrow R\left(T^{k}\right)$ est une bijection. D'où $\operatorname{dim} R\left(T^{k}\right)=\operatorname{dim} X / N\left(T^{k}\right)=\operatorname{codim} N\left(T^{k}\right)<\infty$. Puisque $T^{k}$ est de rang fini, les Théorèmes 11.1 et 11.2 de [14] impliquent qu'il existe un polynôme $P$ non nul tel que $P\left(T^{k}\right)=0$. Si on pose $Q(z)=P\left(z^{k}\right)$ alors $Q$ est un polynôme vérifiant $Q(T)=0$ et donc $T$ est algébrique.

$(4) \Rightarrow(1)$. Soit $T_{0}=T: R\left(T^{j}\right) \rightarrow R\left(T^{j}\right)$. Alors $\operatorname{asc}(T)=j$ implique que $N\left(T_{0}\right)=N(T) \cap R\left(T^{j}\right)=\{0\}$ et donc $T_{0}$ est injectif. D'autre part, $R\left(T^{j+1}\right)$ fermé implique que $T_{0}$ est à image fermée dans $R\left(T^{j}\right)$. Par conséquent, $T_{0}$ est injectif à image fermée dans $R\left(T^{j}\right)$. Or, l'ensemble des opérateurs injectifs à image fermée forme un ouvert, donc il existe $\delta>0$ tel que pour 
tout $|\lambda|<\delta, T_{0}-\lambda I_{0}$ est injectif à image fermée (où $I_{0}=I_{\mid R\left(T^{j}\right)}$ ). D'où $\{0\}=N\left(T_{0}-\lambda I_{0}\right)=N(T-\lambda I) \cap R\left(T^{j}\right)=N(T-\lambda I)$ pour $0<|\lambda|<\delta$. Il résulte alors que $T-\lambda I$ est inversible pour $0<|\lambda|<\delta(\operatorname{car} T \in \mathcal{R}(X))$. Par la continuité de l'indice on déduit que $T_{0}$ est surjectif. Par conséquent, $R\left(T^{j}\right)=R\left(T^{j+1}\right)$, ce qui montre que $\operatorname{desc}(T)<\infty$. Comme en plus, on a $\operatorname{asc}(T)$ finie, donc d'après la proposition 2.8, 0 est un pôle d'ordre fini de la résolvante.

$(1) \Rightarrow(5)$. D'après le Théorème 1.3, il existe $d$ entier tel que $X=N\left(T^{d}\right) \oplus$ $R\left(T^{d}\right)$ avec $\operatorname{dim} R\left(T^{d}\right)<\infty$ (voir la preuve que $(1) \Rightarrow(3)$ ). Soit $P$ la projection sur $N\left(T^{d}\right)$ suivant la décomposition $X=N\left(T^{d}\right) \oplus R\left(T^{d}\right)$. Alors $P$ commute avec $T$ et on a $(T P)^{d}=T^{d} P=0$ et $R(I-P)=R\left(T^{d}\right)$. Maintenant si on pose $F=T(I-P)$ et $Q=T P$ alors $T=Q+F$ avec $Q F=F Q=0$, $Q$ nilpotent et $F$ de rang fini, et (8) est démontré.

$(5) \Rightarrow(2)$. Puisque $Q F=F Q=0, Q$ nilpotent, il existe $d$ entier tel que $T^{d}=F^{d}$. Par conséquent, $\operatorname{dim} R\left(T^{d}\right)=\operatorname{dim} F\left(T^{d}\right)<\infty$, et en reprenant les mêmes arguments que dans $(3) \Rightarrow(2)$, on obtient que $T$ est algébrique.

Remarque. Dans la condition (4) du théorème 2.9, le fait que $R\left(T^{j+1}\right)$ soit fermé est nécessaire, car il existe un opérateur compact dont l'ascente est fini mais la descente non finie (voir [7, Remark 1.3]).

Comme conséquence directe du Théorème 2.9, on obtient la version du Corollaire 2.7, dans l'algèbre de Calkin, suivante :

Corollaire 2.10. Soit $T \in B(X)$ avec $d=\operatorname{desc}(T)$ finie. Alors

$$
\sigma_{e}(T)=\{0\} \Leftrightarrow T \text { est nilpotent dans l'algèbre de Calkin. }
$$

ThÉorème 2.11. Soit $T \in B(X)$ un opérateur méromorphique. Alors les conditions suivantes sont équivalentes:

(1) $0 \in \sigma(T)$ est un pôle d'ordre fini de la résolvente de $T$.

(2) $T$ est algébrique.

(3) Il existe $n \geq 0$ tel que $K(T)=R\left(T^{n}\right)$.

(4) $\operatorname{desc}(T)<\infty$.

(5) $j=\operatorname{asc}(T)$ est finie et $R\left(T^{j+1}\right)$ est fermé.

(6) $T=Q+F$, où $Q, F \in B(X)$, avec $Q F=F Q=0, Q$ nilpotent et $\sigma(F)$ fini.

\section{Références}

[1] P. Aiena, Riesz operators and perturbation ideals, Note Mat. 9 (1989), 1-27.

[2] P. Aiena and M. Mbekhta, Characterisation of some classes of operators by means of the Kato decomposition, Boll. Un. Mat. Ital. A (7) 10-A (1996), 609-621.

[3] S. R. Caradus, Operators of Riesz type, Pacific J. Math. 18 (1966), 61-71. 
[4] S. R. Caradus, W. E. Pfaffenberger and B. Yood, Calkin Algebras and Algebras of Operators on Banach Spaces, Lecture Notes in Pure and Appl. Math. 9, Dekker, New York, 1974.

[5] W. Gong and L. Wang, Mbekhta's subspaces and a spectral theory of compact operators, preprint.

[6] S. Grabiner and J. Zemánek, Ascent, descent, and ergodic properties of linear operators, J. Operator Theory 48 (2002), 69-81.

[7] J. J. Koliha, Isolated spectral points, Proc. Amer. Math. Soc. 124 (1996), 3417-3424.

[8] K. B. Laursen and M. M. Neumann, An Introduction to Local Spectral Theory, London Math. Soc. Monogr. 20, Oxford Univ. Press, 2000.

[9] M. Mbekhta, Ascente, descente et spectre essentiel quasi-Fredholm, Rend. Circ. Mat. Palermo 46 (1997), 175-196.

[10] —, Généralisation de la décomposition de Kato aux opérateurs paranormaux et spectraux, Glasgow Math. J. 29 (1987), 159-175.

[11] M. Mbekhta et A. Ouahab, Opérateur s-régulier dans un espace de Banach et théorie spectrale, Acta Sci. Math. (Szeged) 59 (1994), 525-543.

[12] - - - Perturbation des opérateurs s-réguliers, dans : Topics in Operator Theory, Operator Algebras and Applications (Timişoara, 1994), Rom. Acad., Bucharest, 1995, 239-249.

[13] F. Riesz et B. Sz.-Nagy, Leçons d'analyse fonctionnelle, 4ème éd., Gauthier-Villars et Akadémiai Kiadó, 1965.

[14] Ch. Schmoeger, On isolated points of the spectrum of a bounded linear operator, Proc. Amer. Math. Soc. 117 (1993), 715-719.

[15] A. E. Taylor and D. C. Lay, Introduction to Functional Analysis, Wiley, 1980.

[16] T. T. West, The decomposition of Riesz operators, Proc. London Math. Soc. (3) 16 (1966), 737-752.

[17] -, Decomposition of index-zero Fredholm operators, Proc. Roy. Irish Acad. Sect. A 81 (1981), 49-54.

UFR de Mathématiques

UMR-CNRS 8524

Université Lille 1

59655 Villeneuve d'Ascq, France

E-mail: Widad.Bouamama@agat.univ-lille1.fr

Received March 8, 2002

Revised version April 29, 2003

$(4901)$ 